\title{
Smart Coaster: an example of IoT desing and implementation
}

\author{
Maurizio Rossi, Matteo Nardello and Davide Brunelli \\ University of Trento, Department of Industrial Engineering, 38122 Trento, Italy \\ \{maurizio.rossi, matteo.nardello, davide.brunelli\}@unitn.it \\ https://www.dii.unitn.it/
}

\begin{abstract}
Internet of Things is entering our daily life at a fast pace, with physical devices connected to the internet, collecting and sharing data. In this paper, we describe the design issues of a smart coaster that brings the IoT technology in bars and clubs. The device enables new IoT services and facilities associated to traditional drink glasses. Exploiting this practical example, we present a methodological approach to the IoT design.
\end{abstract}

\section{Introduction}

In this work, we present the design of a smart coaster that has low power consumption, compactness, LoRaWan communication interface and low cost. The system is able to activate itself only when required detecting the fill level of the glass onto it and thanks to wireless recharge, and 3D printed enclosure is cheap and easy to use and maintain. The system has been designed to bring IoT facilities to bars and similar commercial activities, where coasters can interact with the user and notify waiters only when needed, transparently for the customers.

Low-Power Wide Area Network (LPWAN) technologies and LoRaWAN in particular, gained a lot of interests since they hit in the market. LoRa is a wireless telecommunication network that allows long range communication at a very low energy consumption and bit-rate. LoRaWAN stack defines the communication and security protocol that ensures interoperability on top the LoRa network. Many applications have been proposed in literature both in Industry $4.0[2,4]$ and IoT $[3,5,7]$ fields. While LoRa itself is mostly used for ease of customization [6], security and privacy provided by the LoRaWAN stack allows for shorter timeto-market.

\section{Design}

The project has been developed around the $C M W X 1 Z Z A B Z$ SoC developed by Murata [1], because it integrates, in a really small foot print, both the MCU and the LoRa radio. moreover, its ultra-low power consumption, thanks to the use of a STM32L0 MCU, permits to achieve the energy autonomy required by the 

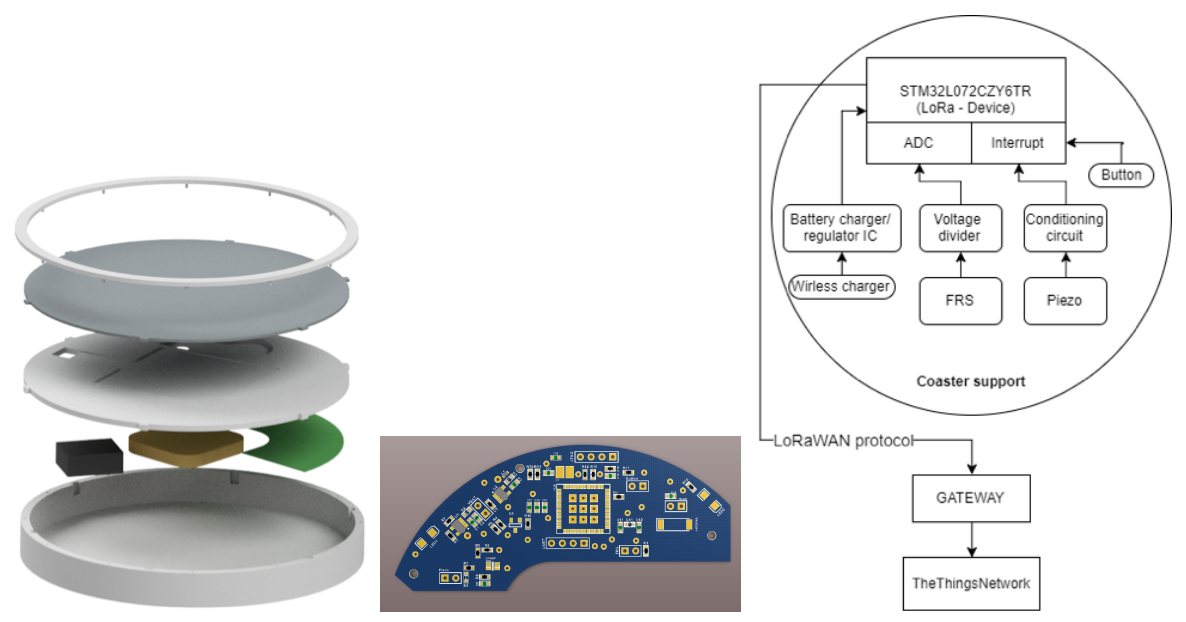

Fig. 1. Coaster 3D design (left), PCB layout (middle) and functional scheme (right).

application. Figure 1-right shows the overall configuration of the system, while figure 1-left presents the final rendering of the prototype.

The system includes multiple interfaces to detect the presence and the state of the glass, a button that can be used by user for calling the waiter and also an inductive charging circuitry used for charging the built-in battery.

\section{D printed coaster}

The physical structure of the coaster is a stacked structure where all the layers can be interlocked on each other, realized using a 3D printer. Starting from the bottom layer up, the first acts as a support, the second support the electronics and the piezo sensors, the third layer is used to achieve uniform pressure distribution over the sensors and the top ring allows the fixing of the structure.

\section{Sensors}

Two kind of sensors were used to detect glass fill level and presence. A Force Sensitive Resistor $(F S R)$ was chosen to detect physical pressure or weight to estimate fill level. This sensor is basically a variable resistor which is composed of two layers of semi-conductive materials separated by a non-conductive spacer. The higher the pressure applied, the lower the resistance of the junction between the two layers. Typically, the resistance varies from $1 \mathrm{M} \Omega$ (unload condition) to some hundreds of $\Omega$ (maximum load condition).

The transfer function is not linear, moreover the output depends on the sensor's shape (square FSR 406 or circular FSR 402) and how the force is applied. The best detection occurs when the tip (on the bottom side of the glass support) covers all the sensor's surface thus to get a homogeneous pressure. The readout is obtained through a voltage divider directly connected to the built-in 12 bit $\mathrm{ADC}$ of the MCU. 
Figure 2 shows how the output changes with respect to the tip shape $(37 \mathrm{x} 37 \mathrm{~mm}$ vs $18 \mathrm{x} 18 \mathrm{~mm}$ ) using the same FSR 406 sensor. These graphs shows that a larger tip (and sensor) provides better and more stable detection of the glass fill level, providing also a greater stability.
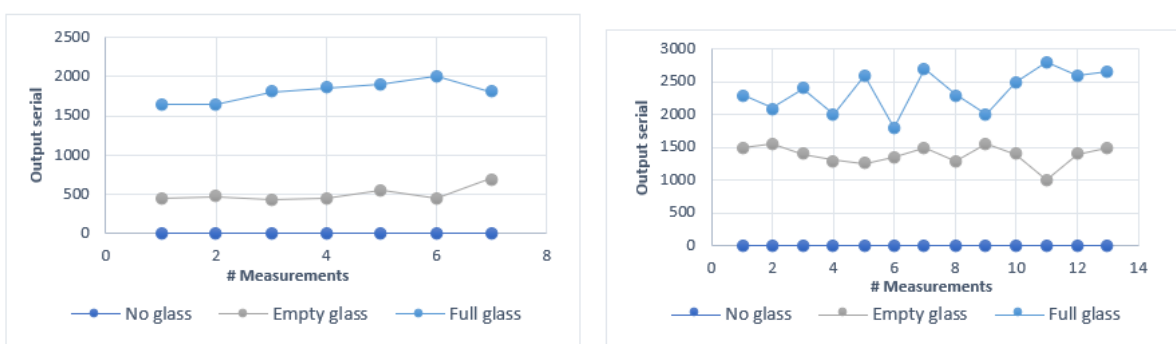

Fig. 2. Response of FSR 406 using $37 \times 37 \mathrm{~mm}$ (left) and 18x18 mm (right) tips.

To detect glass presence while minimizing consumptions, we included a selfpowered wake-up system exploiting a piezo-electric disc (usually employed in musical instruments). This device allows to translate a dynamic stress into a voltage using a charge pump circuitry with a fast rail-to-rail JFET amplifier. Voltage that can be sufficiently high to wake up the MCU.

Lastly, a micro SPDT button is included to interact with the user. This generates a programmable interrupt that can be, for instance, configured to send a dedicated message like "waitress is needed".

Table 1. Energy requirement computed for the IoT device.

\begin{tabular}{|l|l|l|l|}
\hline \multicolumn{5}{|c|}{ Consumptions data } \\
\hline Parameter & Symbol & Value & Unit \\
\hline Worst consumptions & $C_{\text {worst }}$ & 0.0086 & {$[\mathrm{mAh}]$} \\
\hline Sending time & $T_{\text {send }}$ & 4 & {$[\mathrm{~s}]$} \\
\hline Opamp consumptions & $C_{\text {opamp }}$ & 5 & {$[\mathrm{~mA}]$} \\
\hline \multicolumn{4}{|c|}{ Assumptions } \\
\hline Mean working time & $t_{\text {hour }}$ & 8 & {$[\mathrm{~h}]$} \\
\hline Number of messages sent per day & num day & 500 & {$[\#]$} \\
\hline
\end{tabular}

\section{Battery and Charging System}

To guarantee a working life of at least 2 days - considering a typical usage of 8 hours where the ADC is reading and LoRa is sending messages - we choose 
a $100 \mathrm{mAh}$ LiPo battery (Table 1). The battery, $15 x 18 x 7 \mathrm{~mm}$ to fit the coaster, is charged through an inductive charging coil and a Li-Po charger controller, all embedded in the custom PCB, while a LDO that provides a stable $+3.3 \mathrm{~V}$ supply.

\section{Software}

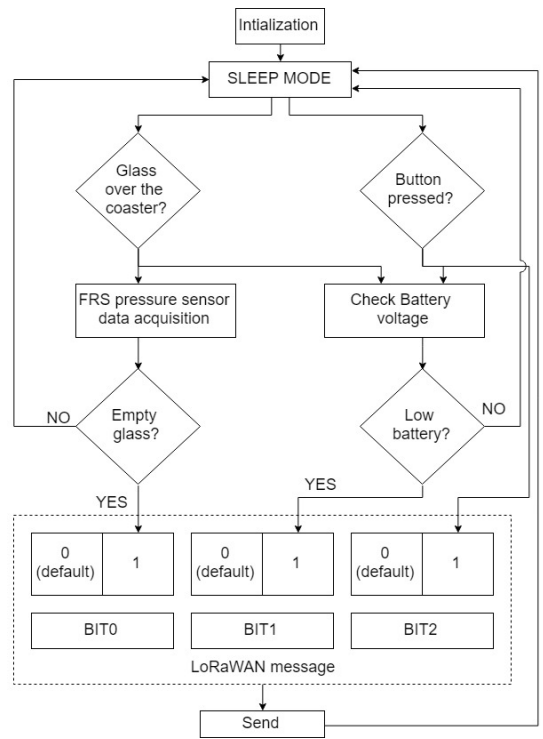

Fig. 3. Firmware flow chart

Initialization: when the micro-controller turns on it configures the necessary registers and peripherals.

Sleep mode: the device remains in sleep mode until an interrupt occurs. This mode reduces consumption but at the same time all peripherals remain active.

Wake up: the device wakes up from the sleep mode when a glass is positioned above it or the user press the call waiter button. In the first case the piezo acts as an interrupt which wakes up the MCU and, after few micro-seconds, enables the ADC reading of the FSR. If the ADC value is in the empty glass range the device sends the message empty glass through the LoRaWAN protocol. In the second case instead, the user can call the waiter just by pressing a button and the device sends the message waiter is needed. For both cases when a message is sent also the battery status (low battery) is sent. After that a led blinks and then the device returns to the sleep mode.

The development of the LoRaWAN protocol on the selected SoC platform has as main characteristics an extremely low CPU load, reduced latencies, small memory footprint, low-power timing services and ensures easy application integration with security and privacy. The implemented network architecture is 
based on the connection of multiple coasters (Class A end devices) with a LoRaWAN gateway implemented on a Raspberry Pi that uses The Things Network as network server. Thanks to a Node-RED instance running on the same gateway it is possible to access all data received. The data package contains the data related to the coaster and the unique coaster- $I D$.
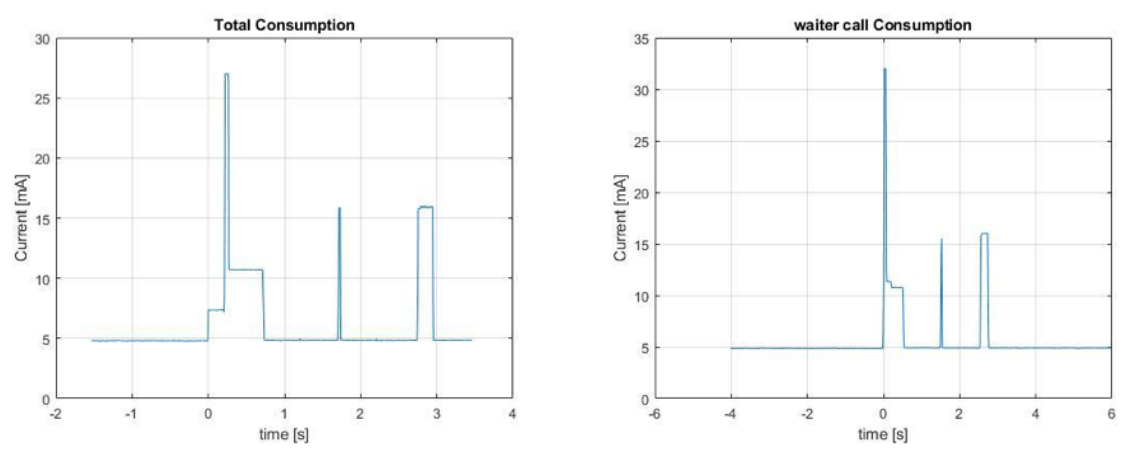

Fig. 4. Current consumption of FSR with LoRa tasks (left) and waiter call with LoRa (right).

\section{Power Consumption}

In this section it is provided an analysis of power consumptions. A shunt resistor and oscilloscope were used to gather the data presented in the following. The first graph (figure 4-left) shows the current drawn when the MCU reads the weight over the coaster and then sends the message, while figure 4-right shows the call waiter operations. In both of these operations there are the LoRa consumptions.

A detail of LoRa consumption is shown in figure 5.

The first highest peak in figure 5 represents the transmission (Tx) followed by two receiving windows ( $\mathrm{Rx}$ ) typical of Class A nodes. During the sleep mode consumptions are approximately $5[\mathrm{~mA}]$, due to the JFET amplifier. For this reason we changed the op-amp with another one with a very low power consumption for the future release. The step after the $T x$ peak, which is present in both graphs of figure 4 but not in figure 5 , is related to the MCU consumption before re-entering the sleep-state. The main difference between the two profiles in figure 4 is related to the fact that when the glass is put on the coaster, left-case, the ADC is read while, during the waiter's call, this doesn't occur.

\section{Conclusions}

In this paper, we presented a smart and compact device able to enclose all the features that make it an innovative and useful coaster with IoT facilities. Main 
design steps have involved the ultra-low-power consumptions, with state-of-theart SoC with built-in LoRaWAN radio and the protocol stack. Finally, built-in power management system with battery and wireless charging.

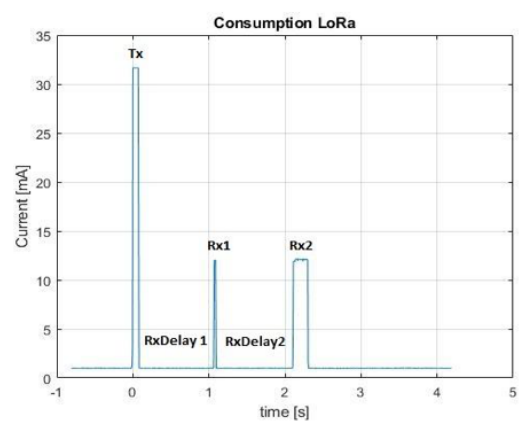

Fig. 5. LoRaWAN consumptions for a class A device

\section{References}

1. CMWX1ZZABZ - wireless.murata.com/RFM/data/type_abz.pdf

2. L. Tessaro, C. Raffaldi, M. Rossi and D. Brunelli, "Lightweight Synchronization Algorithm with Self-Calibration for Industrial LORA Sensor Networks," 2018 Workshop on Metrology for Industry 4.0 and IoT, Brescia, 2018, pp. 259-263. doi: 10.1109/METROI4.2018.8428309

3. G. Dalpiaz, A. Longo, M. Nardello, R. Passerone and D. Brunelli, "A batteryfree non-intrusive power meter for low-cost energy monitoring," 2018 IEEE Industrial Cyber-Physical Systems (ICPS), St. Petersburg, 2018, pp. 653-658. doi: 10.1109/ICPHYS.2018.8390784

4. L. Tessaro, C. Raffaldi, M. Rossi and D. Brunelli, "LoRa Performance in Short Range Industrial Applications," 2018 International Symposium on Power Electronics, Electrical Drives, Automation and Motion (SPEEDAM), Amalfi, Italy, 2018, pp. 1089-1094. doi: 10.1109/SPEEDAM.2018.8445392

5. M. Rizzi, P. Ferrari, A. Flammini and E. Sisinni, "Evaluation of the IoT LoRaWAN Solution for Distributed Measurement Applications," in IEEE Transactions on Instrumentation and Measurement, vol. 66, no. 12, pp. 3340-3349, Dec. 2017. doi: 10.1109/TIM.2017.2746378

6. M. Saravanan, A. Das and V. Iyer, "Smart water grid management using LPWAN IoT technology," 2017 Global Internet of Things Summit (GIoTS), Geneva, 2017, pp. 1-6. doi: 10.1109/GIOTS.2017.8016224

7. P. Neumann, J. Montavont and T. Noël, "Indoor deployment of low-power wide area networks (LPWAN): A LoRaWAN case study," 2016 IEEE 12th International Conference on Wireless and Mobile Computing, Networking and Communications (WiMob), New York, NY, 2016, pp. 1-8. doi: 10.1109/WiMOB.2016.7763213 